7. Lis, M. T., Crampton, R. F., and Matthews, D. M.: Effect of dietary changes on intestinal absorption of L-methionine and L-methionyl-L-methionine in the rat. Br. J. Nutr., 27: 159 (1972).

8. Lis, M. T., Matthews, D. M., and Crampton, R. F.: Effects of dietary restriction and protein deprivation on intestinal absorption of protein digestion products in the rat. Br. J. Nutr., 28: 443 (1972).

9. Matthews, D. M. and Adibi, S. A.: Peptide absorption. Gastroenterology, 71: 151 (1976).

10. Matthews, D. M., Addison, J. M., and Burston, D.: Evidence for active transport of the dipeptide carnosine ( $\beta$-alanyl-L-histidine) by hamster jejunum in vitro. Clin. Sci. Mol. Med., 46: 693 (1974).

11. Matthews, D. M., Gandy, R. H., Taylor, E., and Burston, D.: Influx of two dipeptides, glycylsarcosine and L-glutamyl-L-glutamic acid, into hamster jejunum in vitro. Clin. Sci, 56: 15 (1979).

12. Neame, K. D. and Wiseman, G.: The effect of diet on intestinal active transport. J. Physiol., 146: 10P (1959).

13. Preston, R. L., Schaeffer, J. F., and Curran, P. F.: Structure-affinity relationships of substrates for the neutral amino acid transport system in rabbit ileum. J. Gen. Physiol., 64: 443 (1974).

14. Rubino, A. and Guandalini, S.: Dipeptide transport in the intestinal mucosa of developing rabbits. In: Peptide Transport and Hydrolysis. Ciba Foundation Symposium. Vol. 50, pp. 61-71 (Elsevier Excerpta Medica, New York, 1977).
15. Schedl, H. P., Burston, D., Taylor, E., and Matthews, D. M.: Kinetics of uptake of an amino acid and a dipeptide into hamster jejunum and ileum: the effect of semistarvation and starvation. Clin. Sci., 56: 487 (1979).

16. Steiner, M. and Gray, S. J.: Effect of starvation on intestinal amino acid absorption. Am. J. Physiol., 217: 747 (1969).

17. Sleisenger, M. H., Burston, D., Dalrymple, J. A., Wilkinson, S., and Matthews, D. M.: Evidence for a single common carrier for uptake of a dipeptide and a tripeptide by hamster jejunum in vitro. Gastroenterology, 71: 76 (1976).

18. Sugiyama, K., Yasumoto, K., and Mitsuda, H.: Effect of semi-starvation on kinetic parameters for the in vitro accumulation and transport of L-leucine in rat small intestine. Agric. Biol. Chem., $41: 1969$ (1977)

19. Taylor, E., Burston, D., and Matthews, D. M.: Influx of Glycylsarcosine and L-lysyl-1-lysine into hamster jejunum in vitro. Clin. Sci., 58: 221 (1980).

20. The present address of P. M. Miller is Department of Paediatrics and Child Health, St. James's University Hospital, Leeds, England.

21. Requests for reprints should be addressed to: Dr. M. J. Brueton, Department of Child Health, Westminister Medical School, 146, Vauxhall Bridge Road, London, SW1, England.

22. This research was supported by grants from the National Medical Research Fund, The Frank Odell Charity, Corporate Textiles Ltd., and The Children's Medical Charity.

23. Received for publication April 1, 1983.

24. Accepted for publication August 9,1983

\title{
The Identification and the Excretion Pattern of Isovaleryl Glucuronide in the Urine of Patients with Isovaleric Acidemia
}

\author{
DAVID G. HINE AND KAY TANAKA ${ }^{(14)}$ \\ Department of Human Genetics, Yale University School of Medicine, New Haven, Connecticut, USA
}

\begin{abstract}
Summary
We identified isovaleryl glucuronide in the urine of patients with isovaleric acidemia by using gas chromatography-mass spectrometry (GC-MS) and by identifying the products of enzymatic hydrolysis. Conjugation of isovaleryl-CoA with glycine, by the action of glycine- $\boldsymbol{N}$-acylase, is the main detoxification mechanism in isovaleric acidemia. The identification of isovaleryl glucuronide demonstrates a hitherto unknown, additional detoxification mechanism in patients with isovaleric acidemia. Quantitative analysis of 72 urine specimens from four patients with isovaleric acidemia shows that isovaleryl glucuronide is more likely to be excreted when the amount of urinary 3-hydroxyisovaleric acid excretion is high. This suggests that detoxification via glucuronide conjugation plays an important role when the glycine conjugation system is saturated.
\end{abstract}

\section{Abbreviations}

a.m.u., atomic mass unit

BSA, N,O-bis(trimethylsilyl)acetamide

CI, chemical ionization

EI, electron impact

GC, gas chromatography

MS, mass spectrometry

TMS, trimethylsilyl

Isovaleric acidemia is a recessively inherited disorder of leucine metabolism, resulting from a deficiency of isovaleryl-CoA dehydrogenase $(7,8)$. Clinical manifestations include acute attacks of vomiting, acidosis, ataxia, lethargy, and coma (1, 11). The enzyme block leads to the accumulation of isovaleryl-CoA in tissues. During periods of remission, unoxidized isovaleryl-CoA is handled by conjugation with glycine, catalyzed by the action of glycine- $N$-acylase. Isovalerylglycine thus formed is disposed by urinary excretion (9). When this system is saturated, excess isovaleryl-CoA is hydrolyzed and released as free isovaleric acid causing acute toxic effects. Free isovaleric acid is then omega- 1 oxidized or omega oxidized to 3-hydroxyisovaleric (10) or 4hydroxyisovaleric (13) and methylsuccinic (13) acids, respectively. Small fractions of isovaleryl-CoA are also metabolized to other unusual organic acids including 3-hydroxyisoheptanoic (4) and isovalerylglutamic acid (5).

During the last several years we have identified four patients with isovaleric acidemia by GC and GC-MS. We subsequently analyzed many follow-up samples from these patients. While analyzing these samples, we noticed a well-defined peak which eluted at very high temperatures (methylene unit 22.27 on $10 \%$ $\mathrm{OV}-1$ ) in a number of these samples, in amounts ranging up to $0.50 \mathrm{mg} / \mathrm{mg}$ creatinine. In this report, we describe the identification of this compound as isovaleryl glucuronide using GC and GC-MS as well as enzymatic methods.

\section{MATERIALS AND METHODS}

Chemical materials. "Tri Sil-BSA Formula P" was purchased from Pierce Chemical (Rockford, IL). High purity E. coli $\beta$ - 
glucuronidase type VII was purchased from Sigma Chemical Co. (St. Louis, MO). Coiled glass columns $(2 \mathrm{~mm} \times 6 \mathrm{ft})$ were obtained from Supelco, Inc. (Bellefonte, PA). All other chemicals were reagent grade and obtained from standard commercial sources.

Instrumentation. Gas chromatography was performed using a Hewlett-Packard 5840 gas chromatograph equipped with dual flame ionization detectors and a data system. TMS samples for organic acids were analyzed using a glass column packed with $3 \%$ OV-1 on 100/200 mesh Gas Chrom Q from Applied Science Laboratories (State College, PA). Injection port and detector were kept at $225^{\circ}$ and $300^{\circ} \mathrm{C}$, respectively. The column temperature was programmed from $70-260^{\circ} \mathrm{C}$ at $6^{\circ} \mathrm{C} / \mathrm{min}$. The nitrogen carrier gas flow rate was $25 \mathrm{ml} / \mathrm{min}$. Short chain fatty acid samples were analyzed using a glass column packed with "Carbopack C/O.3\% Carbowax 20M/0.1\% Phosphoric acid" from Supelco, Inc. Injection port and detector were kept at $150^{\circ}$ and $200^{\circ} \mathrm{C}$, respectively. The column temperature was held isothermally at $120^{\circ} \mathrm{C}$. The nitrogen carrier gas flow rate was $25 \mathrm{ml} /$ min.

A Finnigan 4510 GC-MS-COM was used for mass spectral studies. Capillary GC was used as the inlet using a $0.25 \mathrm{~mm} x$ 30 m column coated with DB-5 (SE-54) from J and W Scientific, Inc. (Rancho Cordova, CA). Helium was used as the carrier gas. The split injection mode was used with a flow rate of approximately $1 \mathrm{ml} / \mathrm{min}$. The capillary column temperature was programmed from $70^{\circ}-270^{\circ} \mathrm{C}$ at $5^{\circ} \mathrm{C} / \mathrm{min}$, after an initial hold of 5 min. The injection port, separator, and transfer line were kept at $225^{\circ}, 250^{\circ}$, and $270^{\circ} \mathrm{C}$, respectively. The ionizing voltage was 70 $\mathrm{eV}$ for electron impact analysis and methane was used as reagent gas for chemical ionization analysis.

Sample preparation. Urinary organic acids were analyzed as TMS derivatives as described previously (12). Spot urine samples from patients with isovaleric acidemia were stored at $-20^{\circ} \mathrm{C}$ until analysis.

For short chain fatty acid analysis, an aliquot of urine was adjusted to $\mathrm{pH} 3$ by the addition of $0.5 \mathrm{ml}$ of saturated $\mathrm{K}$ phosphate buffer, $\mathrm{pH}$ 2.90. Five drops of isoamyl alcohol were added as an anti-foaming agent. The sample was transferred to a micro-steam distillation apparatus. Ten milliliters of distillate was collected per $1 \mathrm{ml}$ of sample. The $\mathrm{pH}$ of the distillate was adjusted to $>10$ by the addition of $0.5 \mathrm{ml}$ of $0.1 \mathrm{~N} \mathrm{NaOH}$. The sample was evaporated to dryness with a nitrogen stream in a water bath at $100^{\circ} \mathrm{C}$. One-tenth milliliter of $10 \%$ formic acid was added to redissolve the residue and adjust the $\mathrm{pH}$ to $<3$. One microliter of the sample was injected into the GC.

Hydrolysis of isovaleryl glucuronide with $\beta$-glucuronidase. Preweighed vials containing $1000 \mathrm{U}$ of high purity $\beta$-glucuronidase type VII from $E$. coli were used. When reconstituted with $10 \mathrm{ml}$ of water the solution contained $4 \mathrm{mM}$ phosphate buffer $\mathrm{pH} 6.8$. One milliliter of the enzyme solution was added to $1 \mathrm{ml}$ of urine. As a control, $1 \mathrm{ml}$ of $4 \mathrm{mM}$ phosphate buffer, $\mathrm{pH} 6.8$, was added to another equal aliquot of the same urine. The samples were incubated in a water bath at $37^{\circ} \mathrm{C}$ for $1 \mathrm{~h}$. After incubation the samples were divided into two equal aliquots. One aliquot was extracted with ethyl acetate for organic acid analysis as TMS derivatives and the other aliquot was steam distilled for short chain fatty acid analysis.

\section{RESULTS AND DISCUSSION}

Gas chromatographic detection and mass spectral identification. A typical GC chromatogram of the urine from a patient with isovaleric acidemia, which contained an unidentified, late eluting peak, is shown in Figure 1. This chromatogram was from analysis performed by our routine screening method using a packed $10 \%$ OV-1 column. This sample was subsequently analyzed by GC-MS-COM using a DB-5 capillary column, and the $\mathrm{EI}$ and $\mathrm{Cl}$ spectra obtained are shown in Figures 2 and 3, respectively. The majority of the ions in the EI spectra, with the exception of those at $\mathrm{m} / \mathrm{e} 551,85$, and 57 , are characteristic of
ISOVALERIC ACIDEMIA

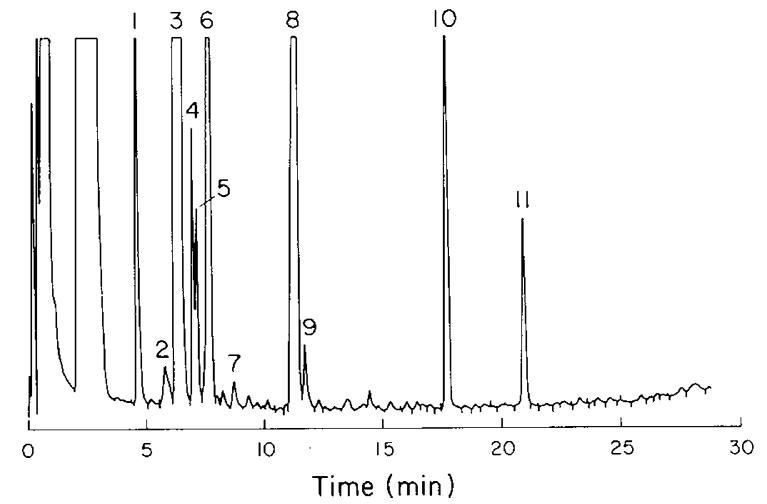

Fig. 1. Gas chromatogram (GC) of an isovaleric acidemia urine sample. A $10 \%$ OV-1 column was used for analysis. The numbered GC peaks were identified by GC-mass spectrometry as: 1) lactic acid; 2) 2-hydroxybutyric acid; 3) 3-hydroxybutyric acid; 4) acetoacetic acid (peak 1); 5) 3-hydroxyisovaleric acid; 6) acetoacetic acid (peak 2), 7) succinic acid; 8) isovalerylglycine; 9) adipic acid; 10) pentadecanoic acid (internal standard); and 11 ) isovaleryl glucuronide.

glucuronide conjugate TMS derivatives (6). These ions characteristic for glucuronides are the ions at $\mathrm{m} / \mathrm{e} 464,449,375,359$, $333,319,305,257,217,204,169,147,129,117,103$, and 95 (6). Glucuronide conjugate TMS derivatives usually do not produce a molecular ion in $\mathrm{EI}$ and frequently fail to do so in $\mathrm{CI}$ as well. We therefore suspected that the ion at m/e 551 corresponded to $M-15$. The $C I$ spectra supports this assumption because the ion at $\mathrm{m} / \mathrm{e} 551$ is of higher intensity and is not shifted 1 a.m.u. higher as would be if it were the molecular ion. For comparison, we ran another sample which was known to contain di(propyl)acetate (valproate) glucuronide TMS derivative under the same $\mathrm{CI}$ conditions and this compound also produced an M-15 ion and no molecular ion. Ions at m/e 85 and 57 are prominent in the EI spectra of the unknown. Given the biochemical nature of the disease and the general fragmentation pattern of glucuronide conjugates we postulated that the ion at $\mathrm{m} / \mathrm{e} 85$ corresponded to an isovaleryl group and the ion at $\mathrm{m} / \mathrm{e} 57$ corresponded to the isobutyl portion of the isovaleryl group as illustrated in Figure 4. The molecular weight of isovaleryl glucuronide tetra-TMS is 566 and hence the ion at m/e 551 would correspond to M-15 as suspected; thus, we identified the unknown as isovaleryl glucuronide based on the mass spectral analysis.

Studies with enzymatic hydrolysis. In order to confirm the identification of isovaleryl glucuronide, we analyzed an isovaleric acidemia urine sample which was pretreated with highly purified $\beta$-glucuronidase from $E$. coli for short chain fatty acids and organic acids as TMS derivatives. This sample contained 150 $\mu \mathrm{g} / \mathrm{ml}(0.54 \mu \mathrm{mol} / \mathrm{ml})$ of isovaleryl glucuronide as determined by GC. As a control we analyzed another aliquot of the same urine that had been treated with the same phosphate buffer alone. The peak identified as isovaleryl glucuronide was unchanged in the sample treated with buffer alone but it was absent in the sample treated with $\beta$-glucuronidase. Otherwise, the organic acid profile including isovalerylglycine was essentially identical quantitatively, indicating high specificity of glucuronidase action. The chromatograms obtained for short chain fatty acid analysis are shown in Figure 5. The amount of isovaleric acid greatly increased in the sample treated with $\beta$-glucuronidase. The identity of isovaleric acid was confirmed by coinjection of authentic isovaleric acid. The additional isovaleric acid liberated by glucuronidase treatment was calculated to be $67 \mu \mathrm{g} / \mathrm{ml}(0.66$ $\mu \mathrm{mol} / \mathrm{ml}$ ) which is $22 \%$ higher than the estimated amount of isovaleryl glucuronide. This small discrepancy was determined to be due to the fact that isovaleryl glucuronide was not quantitatively recovered by ethyl acetate extraction which was utilized 


\section{EI SPECTRUM OF ISOVALERYLGLUCURONIDE FROM PATIENT SAMPLE}
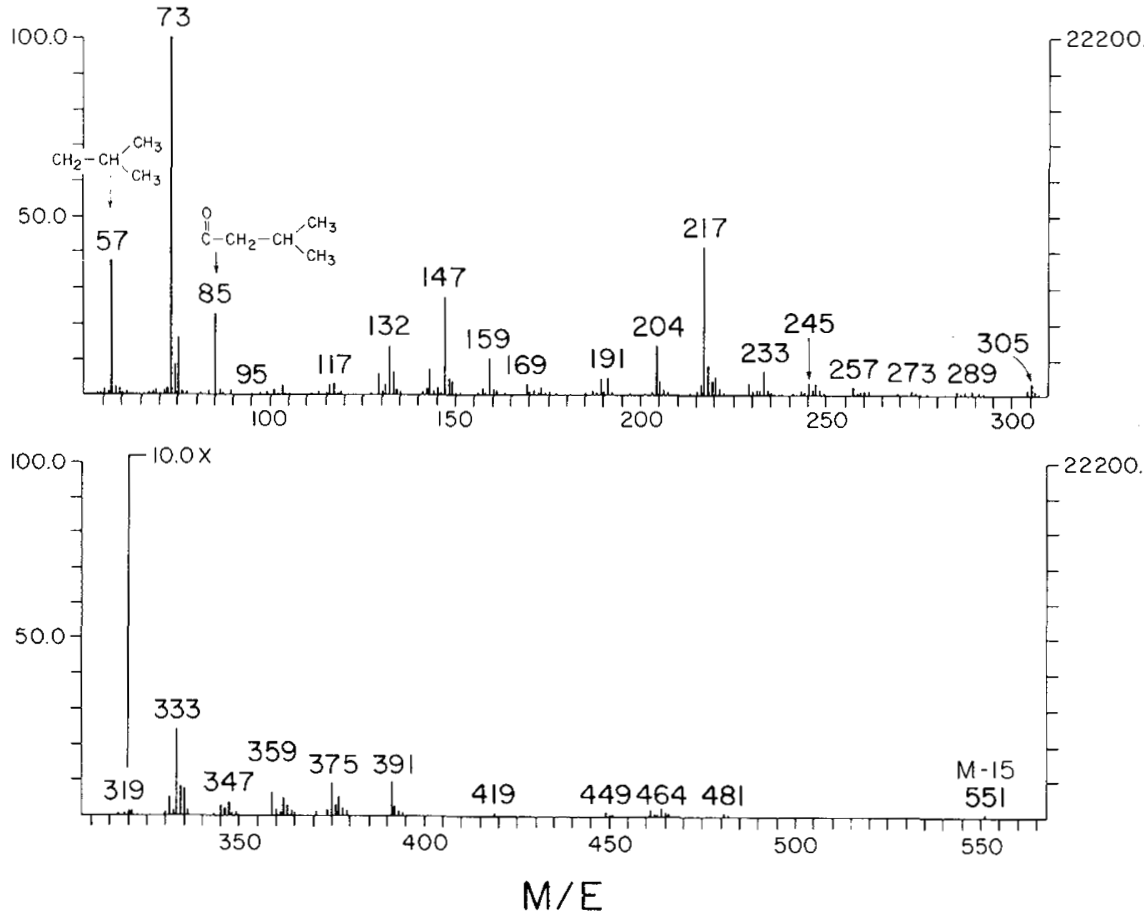

Fig. 2. Electron impact mass spectrum of gas chromatography peak \#11 from Figure 1.
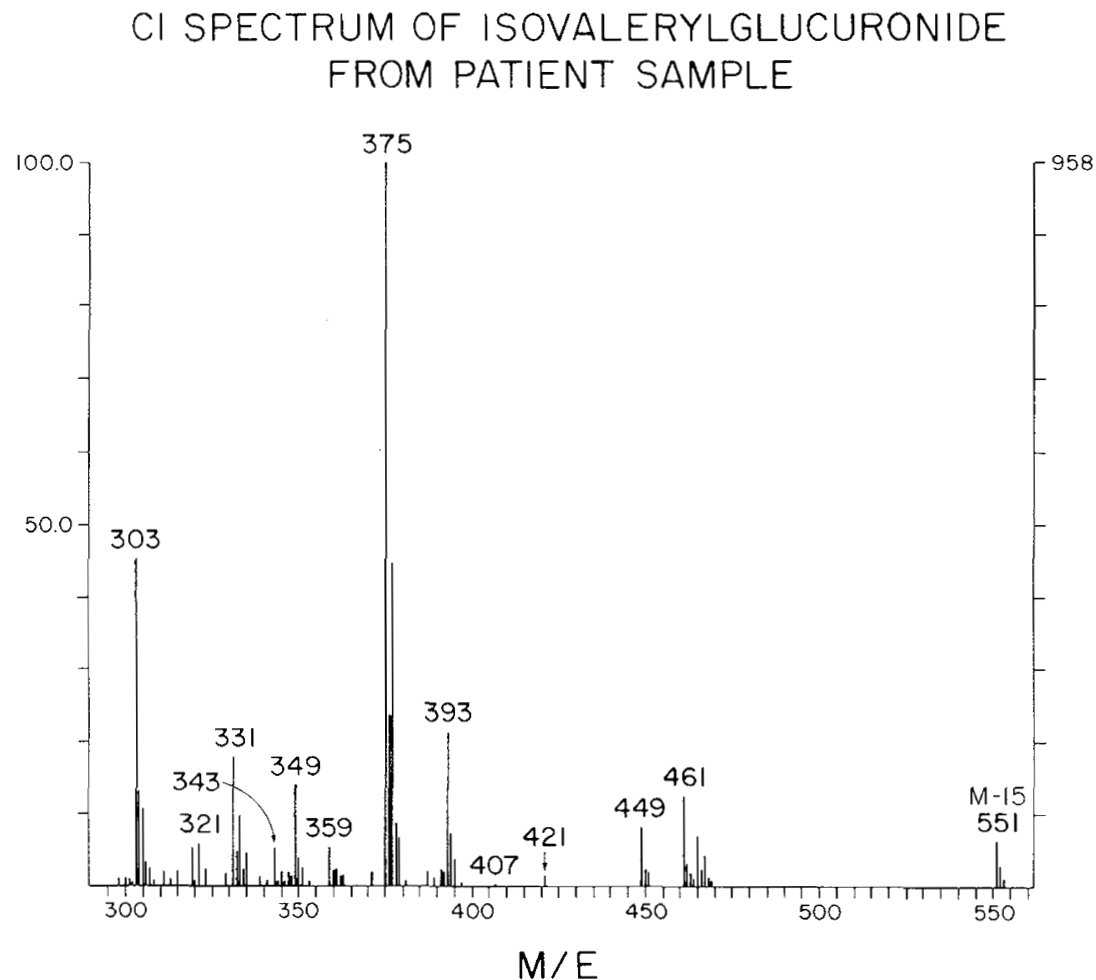

Fig. 3. Chemical ionization mass spectrum of gas chromatography peak \#11 from Figure 1.

in our organic acid TMS method. Significant amounts of isovaleryl glucuronide $(20-30 \%)$ were detected in the lyophilized aqueous phase after four extractions with ethyl acetate.

Some investigators have questioned the selectivity of $\beta$-glucuronidase and have speculated that the enzyme may also cleave glycine conjugates (3). In order to rule out isovalerylglycine as the source of the isovaleric acid liberated by glucuronidase treat- ment of the urine sample, we incubated authentic isovalerylglycine $(1 \mu \mathrm{mol} / \mathrm{ml})$ with the same $\beta$-glucuronidase preparation. We detected no release of free isovaleric acid, excluding hydrolysis of isovalerylglycine by this enzyme.

In order to identify glucuronic acid in the $\beta$-glucuronidasetreated urine, we acidified the urine sample and extracted several times with ethyl acetate. Glucuronic acid is insoluble in organic 
M.W. $=566$

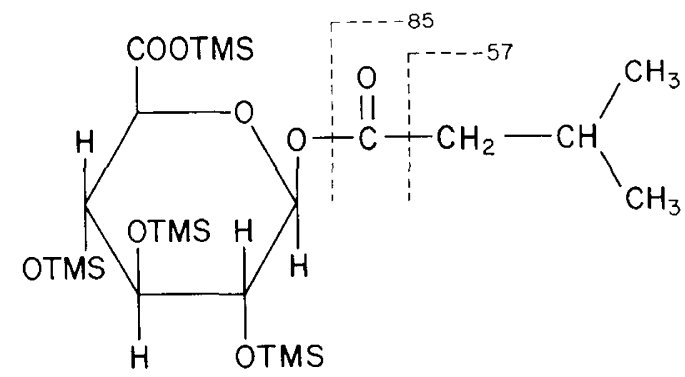

Fig. 4. Structure of isovaleryl glucuronide tetra-TMS.

SHORT CHAIN FATTY ACIDS

\section{A. Untreated Urine}

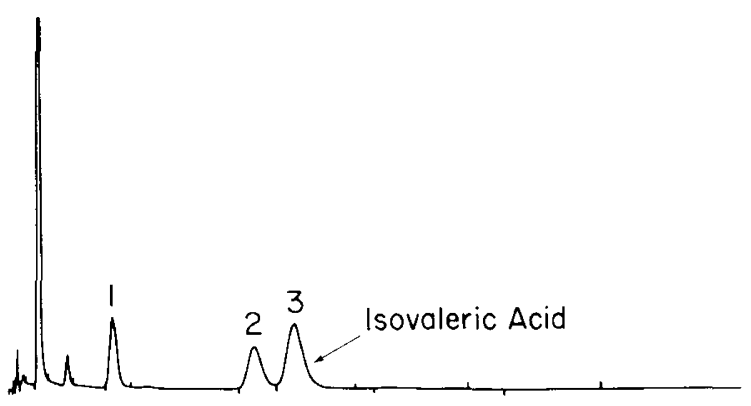

B. Glucuronidase-treated Urine

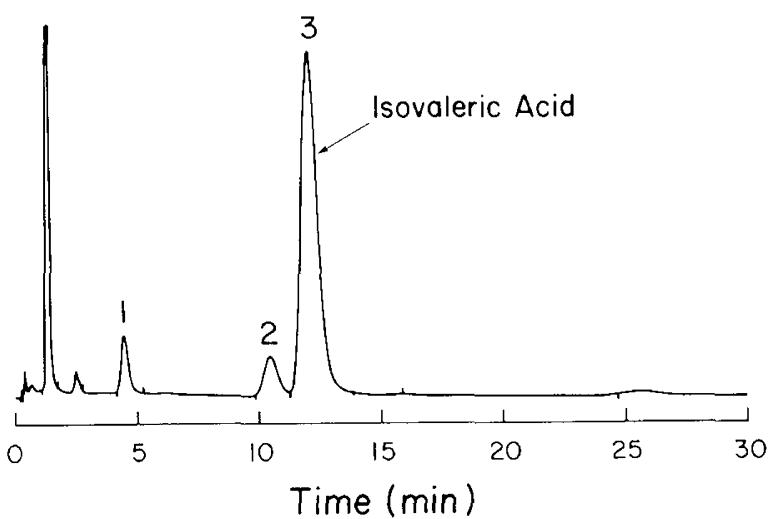

Fig. 5. Short chain fatty acid gas chromatograms $(\mathrm{GC}): A$ ) an isovaleric acidemia urine sample treated with buffer alone and $B$ ) the same isovaleric acidemia urine sample treated with $E$. coli $\beta$-glucuronidase. The numbered GC peaks were identified as: $l$ ) isobutyric acid; 2) 2methylbutyric acid; and 3) isovaleric acid.

solvents and remained in the water layer. The water layer was lyophilized and the dried residue was silylated with Tri-Sil BSA. A well-defined peak which elutes before the glucuronide conjugate (methylene unit $20.88,3 \% \mathrm{OV}-1$ ) was positively identified as glucuronic acid penta-TMS by GC-MS by matching the spectrum obtained to that of authentic glucuronic acid pentaTMS. The peak obtained was smaller than might have been expected. We also carried out a similar experiment using authentic phenolphthalein glucuronide. The recovery of glucuronic acid from the phenolphthalein glucuronide hydrolyzate by the same $\beta$-glucuronidase was similar to that observed in the experiment using the urine of patients with isovaleric acidemia. The failure of quantitative recovery of glucuronic acid may be due partly to the fact that glucuronic acid also forms various lactones (2).

Urinary excretion pattern. We have identified isovaleryl glucuronide in the urine of patients with isovaleric acidemia. This is the first time that detoxification via glucuronide conjugation has been demonstrated in an inborn error of metabolism. The glucuronide conjugation of isovaleric acid is probably an important aspect of the detoxification mechanisms in isovaleric acidemia.

In order to ascertain the precipitating factors which lead to the excretion of isovaleryl glucuronide, we reviewed 72 chromatograms from four patients with isovaleric acidemia. We suspected that glucuronide conjugation may become activated when the glycine conjugation system is saturated. When the glycine conjugation system is saturated, free isovaleric acid is oxidized to 3hydroxyisovaleric acid, which appears in the urine (10). We examined the excretion pattern of 3-hydroxyisovaleric acid, isovaleryl glucuronide, and isovalerylglycine as shown in Tables 1 and 2. The peaks for the three metabolites were identified using methylene unit valves compiled on $10 \% \mathrm{OV}-1$ and $10 \% \mathrm{OV}-17$ packed columns (12). The homogeneity of the gas chromatogram

Table 1. Urinary excretion of isovaleryl glucuronide and isovalerylglycine as related to the amount of 3-hydroxyisovaleric acid (values expressed as $\mathrm{mg} / \mathrm{mg}$ creatinine)

\begin{tabular}{ccc}
\hline $\begin{array}{c}\text { 3-Hydroxyisovaleric } \\
\text { acid }\end{array}$ & Isovaleryl glucuronide & Isovaleryglycine \\
\hline $0(n=49)^{*}$ & $0(n=31)-0.54$ & $1.07-12.27$ \\
0.04 & 0 & 2.77 \\
0.04 & 0 & 6.72 \\
0.05 & 0 & 3.88 \\
0.07 & 0 & 3.80 \\
0.07 & 0.04 & 4.70 \\
0.07 & 0 & 5.51 \\
0.07 & 0.03 & 6.29 \\
0.08 & 0.04 & 1.56 \\
0.08 & 0 & 3.11 \\
0.08 & 0 & 5.02 \\
0.08 & 0 & 5.51 \\
0.09 & 0.08 & 3.31 \\
0.16 & 0.45 & 5.28 \\
0.26 & 0 & 5.87 \\
0.26 & 0 & 11.90 \\
0.30 & 0.02 & 5.02 \\
0.34 & 0 & 6.60 \\
0.38 & 0.05 & 1.07 \\
0.39 & 0.04 & 5.49 \\
0.77 & 0.07 & 8.46 \\
0.88 & 0.23 & 5.84 \\
0.90 & 0.25 & 5.70 \\
1.00 & 0.10 & 7.74 \\
\hline
\end{tabular}

* The number of samples are indicated in parenthesis.

Table 2. The amounts of 3-hydroxyisovaleric acid and isovalerylglycine in urine samples containing, and not containing, isovalerylglucuronide

\begin{tabular}{|c|c|c|c|}
\hline & $\begin{array}{l}\text { Number of } \\
\text { samples }\end{array}$ & $\begin{array}{c}\text { 3-Hydroxyisoval- } \\
\text { eric } \\
\text { acid* }^{*} \\
\text { (mg/mg creatinine) }\end{array}$ & $\begin{array}{c}\text { Isovalerylglycine } \\
\text { (mg/mg creatinine) }\end{array}$ \\
\hline $\begin{array}{l}\text { Samples con- } \\
\text { taining isoval- } \\
\text { eryl glucuro- } \\
\text { nide }\end{array}$ & 29 & $0.176 \pm 0.312 \dagger$ & $4.38 \pm 1.90$ \\
\hline $\begin{array}{l}\text { Samples con- } \\
\text { taining no iso- } \\
\text { valeryl glucu- } \\
\text { ronide }\end{array}$ & 43 & $0.032 \pm 0.076$ & $4.70 \pm 2.62$ \\
\hline
\end{tabular}

* Twenty-three samples contained 3-hydroxyisovaleric acid. $\dagger$ Values given as mean $\pm 1 \mathrm{SD}$. 
peaks was confirmed, in each case, for five randomly selected samples which were analyzed by GC-MS. Although there is no linear, quantitative correlation between the amounts of 3-hydroxyisovaleric acid and isovaleryl glucuronide, it is apparent that isovaleryl glucuronide is more likely to be excreted when the amount of 3-hydroxyisovaleric acid is high. For example, as can be seen in Table 1, when the amount of 3-hydroxyisovaleric acid was greater than $0.26 \mathrm{mg} / \mathrm{mg}$ creatinine, isovaleryl glucuronide was present in seven out of eight samples. When the amount of 3-hydroxyisovaleric acid was equal to or less than $0.26 \mathrm{mg} / \mathrm{mg}$ creatinine, isovaleryl glucuronide was present in only 23 of 64 samples. Table 2 shows that the average amount of 3-hydroxyisovaleric acid was more than five times greater in those samples that contained isovaleryl glucuronide, whereas the amount of isovalerylglycine was roughly equal in both groups. The data therefore does indicate that glucuronide conjugation of isovaleric acid is more prevalent when the glycine conjugation system is saturated. It should be noted, however, that of the 49 samples which contained no measurable amount of 3-hydroxyisovaleric acid, 18 contained isovaleryl glucuronide in varying amounts ranging up to $0.54 \mathrm{mg} / \mathrm{mg}$ creatinine (see Table 1). There may be other factors involved in the stimulation of the glucuronide detoxification mechanism. Nevertheless, glucuronide conjugation appears to be assuming the role of a secondary, yet important, detoxification mechanism in isovaleric acidemia.

\section{REFERENCES AND NOTES}

1. Budd, M. A., Tanaka, K., Holmes, L. B., Efron, M. L., Crawford, J. D., and Isselbacher, K. J.: Isovaleric aciemia: clinical features of a new genetic defect of leucine metabolism. N. Engl. J. Med., 277 (1976).

2. Dutton, G. J.: Glucuronic Acid, Free and Combined. (Academic Press, New York, 1966).

3. Fenselau, C. and Johnson, L.: Analysis of intact glucuronides by mass-spec- trometry and gas chromatography-mass spectrometry. Drug Metab. Dispos., 8: 274 (1980).

4. Lehnert, W.: 3-Hydroxyisoheptanoic acid: a new metabolite in isovaleric acidemia. Clin. Chim. Acta, 113: 101 (1981)

5. Lehnert, W.: Excretion of $\mathrm{N}$-isovalerylglutamic acid in isovaleric acidemia. Clin. Chim. Acta, 116: 249 (1981)

6. Mrochek, J. E. and Rainey, W. T.: Gas chromatography and mass spectrometry of some trimethylsilyl derivatives of glucuronide. Anal. Biochem., 57: 173 (1974).

7. Rhead, W. R. and Tanaka, K. Demonstration of a specific mitochondrial isovaleryl CoA dehydrogenase deficiency in fibroblasts from patients with isovaleric acidemia. Proc. Natl. Acad. Sci. U.S.A., 77: 580 (1980).

8. Tanaka, K., Budd, M. A., Efron, M. L., and Isselbacher, K. J.: Isovaleric acidemia: a new genetic defect of leucine metabolism. Proc. Natl. Acad. Sci. U.S.A., 56: 236 (1966).

9. Tanaka, K. and Isselbacher, K. J.: The isolation and identification of Nisovalerylglycine from urine of patients with isovaleric acidemia. J. Biol. Chem., 242: 2966 (1967).

10. Tanaka, K., Orr, J. C., and Isselbacher, K. J.: Identification of 3-hydroxyisovaleric acid in the urine of a patient with isovaleric acidemia. Biochem. Biophys. Acta, 152: 638 (1968).

11. Tanaka, K. and Rosenberg, L. E.: Disorders of branched chain amino acid and organic acid metabolism. In: J. B. Stanbury, J. B. Wyngaarden, D. S. Fredrickson, J. L. Goldstein, and M. S. Brown: The Metabolic Basis of Inherited Disease. (McGraw-Hill, New York, 1982).

12. Tanaka, K., West-Dull, A., Hine, D. G., Lynn, T. B., and Lowe, T.: Gas chromatographic method of analysis for urinary organic acids. II. Description of the procedure, and its application to diagnosis of patients with organic acidemia's. Clin. Chem. 26:1847 (1980).

13. Truscott, R. J. W., Malegan, D., McCairs, E., Burke, D., Hick, L., Sims, P. Halpern, B., Tanaka, K., Sweetman, L., Nyhan, W. L., Hammond, J., Bumack, C., Haan, E. A., and Danks, D. M.: New metabolites in isovaleric acidemia. Clin. Chim. Acta, 110:187 (1981).

14. Requests for reprints should be addressed to: Kay Tanaka, M.D., Yale University School of Medicine, Department of Human Genetics, 333 Cedar Street, P.O. Box 3333, New Haven, Connecticut, 06510, (USA).

15. We would like to thank Ms. Kelly Beckman for her assistance in the preparation of this manuscript.

16. Received for publication April 19, 1983.

17. Accepted for publication August 10, 1983.

\title{
Colostrum-Induced Enteric Mucosal Growth in Beagle Puppies
}

\author{
WILLIAM C. HEIRD, ${ }^{(27)}$ STEVEN M. SCHWARZ, AND INGE H. HANSEN \\ Department of Pediatrics and Institute of Human Nutrition, Columbia University College of Physicians and \\ Surgeons, New York, New York, USA
}

\section{Summary}

To evaluate the role of artificial feeding and natural feeding in early growth of enteric mucosa, we determined enteric mucosal mass, protein and DNA content, and disaccharidase activities in beagle puppies at birth, and after $24 \mathrm{~h}$ of either natural or artificial feeding. Despite similar increases in body weight over the first $24 \mathrm{~h}$ of life, neither mucosal mass, DNA content, nor protein content of the artificially fed animals was different from that of newborn animals. In contrast, mucosal mass of the suckled animals was $75 \%$ greater, DNA content was $56 \%$ greater, and protein content was $93 \%$ greater than that of newborn animals. The mucosal protein/DNA ratio of the suckled animals was greater than that of newborn, but not artifically fed animals. The greater DNA, protein, and protein/DNA ratio in this group suggest that the greater mucosal mass is a result of both cellular hyperplasia and hypertrophy. Sucrase specific activity of the suckled animals was less than that of the artificially fed but not the newborn animals. Other disaccharidase activities were not different among the three groups.

These data extend the findings of Widdowson $e t$ al. (25) to another species and demonstrate that this rapid enteric growth over the first day of life results only from natural feeding. They strongly suggest, therefore, that rapid early enteric growth, mediated perhaps by a factor in natural milk that stimulates enteric mucosal growth, is an important heretofore unappreciated phase of intestinal development.

In contrast to the well described alterations that take place immediately after birth in many organ systems (i.e., the cardiovascular and respiratory symptoms), very little is known about 